\title{
Study on Power Control in the Cognitive AD HOC Self-organizing Network
}

\author{
Wang Ting \\ School of Electronic and Information Engineering, Lanzhou Jiao Tong University, \\ Lanzhou 730070, China
}

\begin{abstract}
Nodes in ad hoc networks form dynamic topologies typically with scarce resources, such as energy and bandwidth. A main challenge in the operation of such networks is the efficient allocation of resources for radio communications. In this context, the presented paper proposes a power control mechanism created as a network-assisted function of ad hoc cognitive packet-based routing and aimed at reducing both energy consumption in nodes and mutual interference of adjacent communications. Simulation results in NS-2 show significant energy savings after applying the proposed algorithm. In addition, simulation results and a mathematical analysis of interference models, depict a reduction of neighboring radio interference and results in a promising increase of network throughput.
\end{abstract}

Keywords: Cognitive Radio; Ad Hoc Networks; Power Control; Routing Protocol

\section{Introduction}

Mobile Ad Hoc networks (MANET) are self-organizing networks, which are composed of mobile nodes and with dynamic topological structures. There is no fixed infrastructure in the network; the mobile nodes are the communication agents, and also have the function of packet forwarding. In addition to acting as end systems, nodes in ad hoc networks also serve as transit nodes of other communication links. Their participation in the process of searching for paths (routing) and packets forwarding depends on the availability of internal resources. Due to the specific characteristics of mobile nodes, these resources are always scarce.

Ad hoc cognitive packet networks (AHCPN) [1] is a novel Ad hoc network routing protocol. Cognitive packet network (CPN) [2-5] is a fast adaptive routing algorithm that exploits learning to discover and refine routes. The creation and maintenance of routes are realized by cognitive or smart packets (SP), which are sent out by source nodes when new destinations are needed. With the SPs moving in the network to collect information and to make decisions, what previous packets have learned has to be considered. Decisions needed to be tailored to reflect a desired quality of service (QoS) on the path, for example, to minimize end-to-end delay or power consumption. In-depth studies of other ad hoc routing protocols can be found in [6].

\section{Power-aware Routing Protocols}

Singh, [7], studied power efficiency as well as routing protocols, and investigated the power-aware metrics employed in shortest path calculation. And the power demand of packets sending and receiving on the links are measured; the methods to minimize power demand of end-to-end routing are explored. However, the algorithm proposed did not consider the residual energy of nodes, which would lead to battery energy depletion of nodes on the shortest path. 
Other existing algorithms take the battery lifetime into account avoiding the problems in [7]. For example, Toh [8] proposed a new measurement method to calculate the summation of remaining battery capacities of nodes along the reverse path. In addition, he puts forwarded the min-max algorithm to maintain a fair use of resources by avoiding the use of nodes with the least remaining-battery capacity in the network. Li et al. [9] propose an algorithm (denoted by max-min zPmin) that calculates the minimal energy consumption on the path to maximize the minimal residual power of the network.

Power-aware source routing (PSR) [10] is similar with DSR, but the destination calculates the link cost based on the remaining battery capacity and transmission power of the nodes. The drawback of this approach is that the destination needs to wait for some time since the arrival of the first route request, so as to receive more than one possible route and then to select one with the minimum cost.

Another feasible approach is employed by AFA (adaptive fidelity algorithm) [11], which is based on the on-demand ad hoc routing protocols, such as AODV and DSR, etc., AFA saves battery power by turning off the transceivers in case that the applications allow for quality reductions of the links. The algorithm trades link quality for battery lifetime, network bandwidth, or the number of active sensors

Kawadia, et al., [12 - 13], propose several power control algorithms, among which the COMPOW and CLUSTERPOW, as two known algorithms, are summarized in this paper. The goal of COMPOW is to reduce the transit power level of a given node to the lowest level with the number of neighbor nodes unchanging; CLUSTERPOW use the minimum transmit power $\mathrm{p}$, and every node forwards a packet for the destination node using a power level less than $\mathrm{p}$ in multichip. Both COMPOW and CLUSTERPOW can reduce power consumption and extend the battery lifetime of the nodes, but they cannot realize other objectives of QoS. The CLUSTERPOW algorithm trades battery lifetime for timelapse.

\section{AD HOC Network Model}

\subsection{Path Loss and Decay}

Path loss is the ratio of transmit power to the receive power, which affects the received signal quality and its function expression is as follows:

$$
P_{r x}=O\left(\frac{P_{t x}}{d^{\alpha}}\right)
$$

In the above expression, the implicit constant in $\mathrm{O}$ depends on carriers' physical properties (i.e., antenna gain and frequency), and $\alpha$ is between 2 and 4. Expression (1) is also called the "path loss model". However, in reality, the environment is not a free space and obstacles (e.g., building, terrain, and environmental characteristics) resulting in reflection, scattering and diffraction, etc. may occur; thus, $\alpha$ varies within 2 to 4 .

\subsection{Interference Model}

For a given node, in addition to path loss, the bit error rate of data sending will also influence the receiving quality. The bit error rate is determined by noise power, transmit power and the relative position of other emitting nodes (that are assumed to transmit using same communication channels).

In this paper, two existing interference models are discussed: physical model and protocol model. 


\subsection{Physical Model}

In the physical interference model, the accumulated power of neighbor nodes being noise, signal-to-noise ratio (SNR) and more accurate signal to interference ratio (SIR) are calculated.

Let $\mathrm{Xi}$ be the simultaneously-sending node group. $\mathrm{Pi}$ is the transmit power of a given node $\mathrm{Xi}$; if the following condition holds, the transmit power of $\mathrm{Xi}$ can be successfully received by $\mathrm{Y}$ :

$\frac{\frac{p_{i}}{d\left(X_{i}, Y\right)^{\alpha}}}{N+\sum \frac{P_{k}}{d\left(X_{k}, Y\right)^{\alpha}}} \geq \beta$

Where $\beta$ is the minimum value of SIR.

\subsection{Protocol Model}

The precondition for the protocol model to work is: the data packets transmitted can be successfully received only if the receive power of the receiver is greater than or equal to those of other transmitters. The packet transmitted by $\mathrm{Xi}$ is successfully received by $\mathrm{Y}$; if for all $\mathrm{k}$, the following condition holds:

$$
\frac{p_{i}}{d\left(X_{i}, Y\right)^{\alpha}} \geq(1+\Delta) \frac{P_{k}}{d\left(X_{k}, Y\right)^{\alpha}}
$$

Where $\Delta$ is the precautionary area of a specific protociols for interference prevention.

\section{Power Control in AHCPN}

In cognitive packet network, three types of packets are used to complete the forwarding function of routers: smart packet (SP), dumb packet (DP), and acknowledgement (ACK). $\mathrm{SP}$ is responsible for route discovery and route maintenance; using the SP source route that can perform route discovery, DP transmits the payload from source node to the destination. Each CPN packet has three elements: head node transmission source address, destination address and the useful information processing of other data packets, e.g., packet length. The cognitive map refers to the area of data packets that are used for network information storage, and only DP uses this data area for payload transmission [1].

\subsection{Unicast Routing}

The execution of the CPN routing decisions is based on a self-organizing neural network, and trained by the reinforcement learning algorithm. AHCPN adopts almost the same decision-making method of unicast routing protocol as CPN except a small difference. CPN replaces a small portion of decisions by random decisions to prevent this algorithm from being trapped in a local minimum. In this paper, AHCPN uses broadcast instead of random decisions to make this algorithm better adapt to the mobile network. Since explanation about this algorithm has been made in [2-5], more information about CPN shall not be given here.

\subsection{Implementation Process of Algorithm}

Using DP source routing datagrams of the storage path in source nodes' route cache, there will be enough energy to reach the destination. And the maximum power is used for SP and SP acknowledgement in this network. On the basis of SP acknowledgement, using this method, SP will try to achieve routing decisions of all nodes within the maximum 
communication range. When the destination of data sending is unknown, and DP searches for route, SP will wait in the queue [14].

Figure 1 shows the data packet generation process on the source node. When the data packets reach and store at the locality, the source node will continuously send smart packets until the destination of source node's route request is found.

The unicast or broadcast is used by SP to transmit on the network, but the method ultimately adopted depends on the information provided by its location. When the information available indicates it cannot be determined whether unicast (RNN-based) should be used, broadcast is the optimal methods (as shown in Figure 2). It is worth noting that at least three neighbor nodes need to adopt the RNN algorithm at random forwarding nodes. A neighbor node simply receive data packets and does not participate in the calculation (split horizon), but the source node needs at least two neighbor nodes.

After the route to new destination is set up, the received signal power will be used to calculate the attenuation of the signal at each jump; and each node will adopt the power which is calculated based on the collected information of SP and SP acknowledgement time. The smart packets may be used to maintain (or improve) link quality, and are only a small portion of dumb packets.

AHCPN will provide the full path of payload transmission (which refers to datagram in this case) for dumb packets. The original data replications will be stored at source nodes until data packets are successfully transmitted to the destination nodes. They are acknowledged by the dumb packets: to confirm data packets transmission and mailbox update on the path to the source node.

In the process of dumb packets transmission on the network, they will update the mailboxes by collecting timestamps, but their acknowledgements require the collection of link quality information on the path.

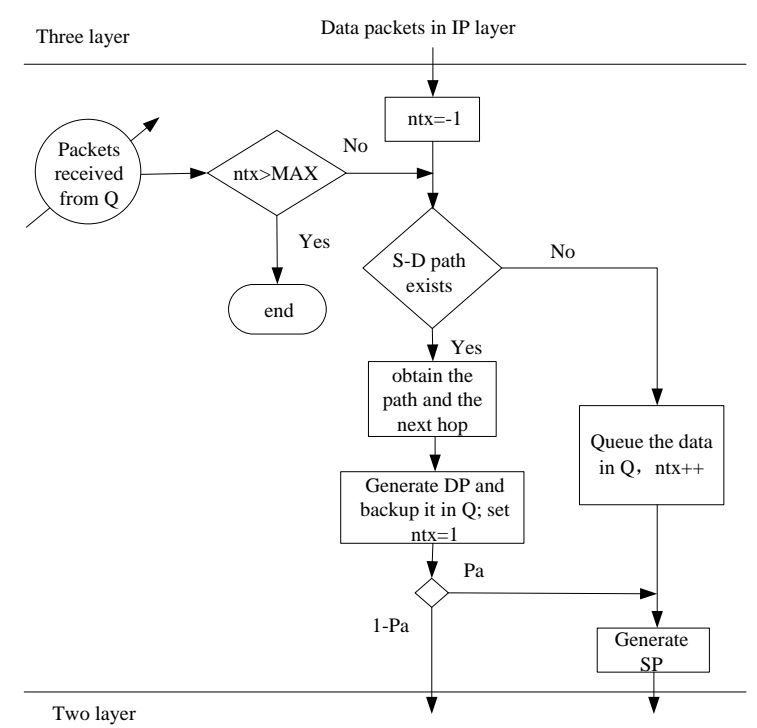

Figure 1. Data Packet Generation at the Source Node 


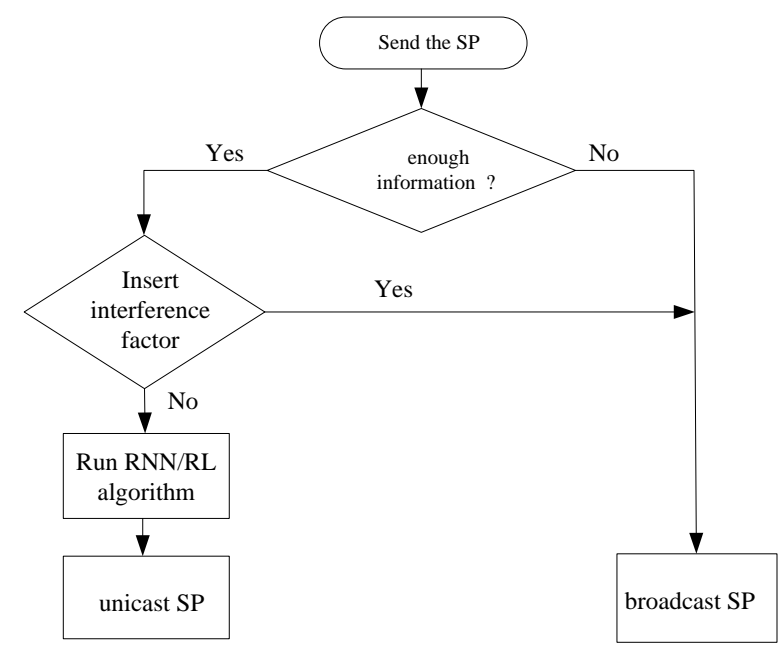

Figure 2. The Decision Logic in Smart Packets

\section{Simulation}

The AHCPN algorithm implementation is developed and integrated in network simulator 2 (NS-2). In this paper, the purpose of the experiment is to examine the establishment and use of route in a network. The runtime of each network simulation is 1000 seconds. According to different power control algorithms adopted, the simulation can be divided into two cases, and the simulation results of 45 nodes are collected. It is supposed that all nodes are static, and Figure 3 shows the topological structure. In the beginning, we assume that all node batteries have been fully charged.

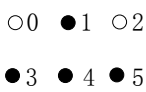

$06 \bullet 7 \bigcirc 8$
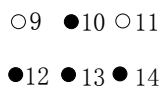

$015 \bullet 16 \bigcirc 17$
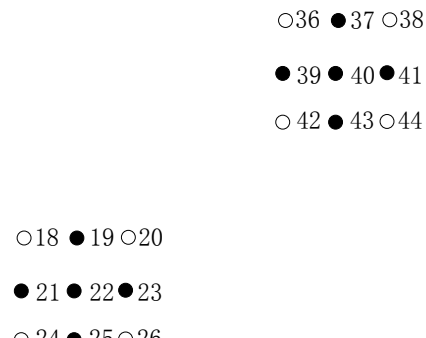

○ 27 • $28 \bigcirc 29$

- $30 \bullet 31 \bullet 32$

○ 33 • 34035

\section{Figure 3. The Simulated Network Topology}

In the simulation, 16 links are set up between corner nodes of the gird and those of the entire network. Node 0 and node 17 also have two data streams in opposite directions, which are added to the communication. According to AHCPN, the bit rate of smart packets transmission is 0.2 (20SP per 100DP). In Section 4, we introduce in detail the objective functions, including the round-trip delay and battery information. 


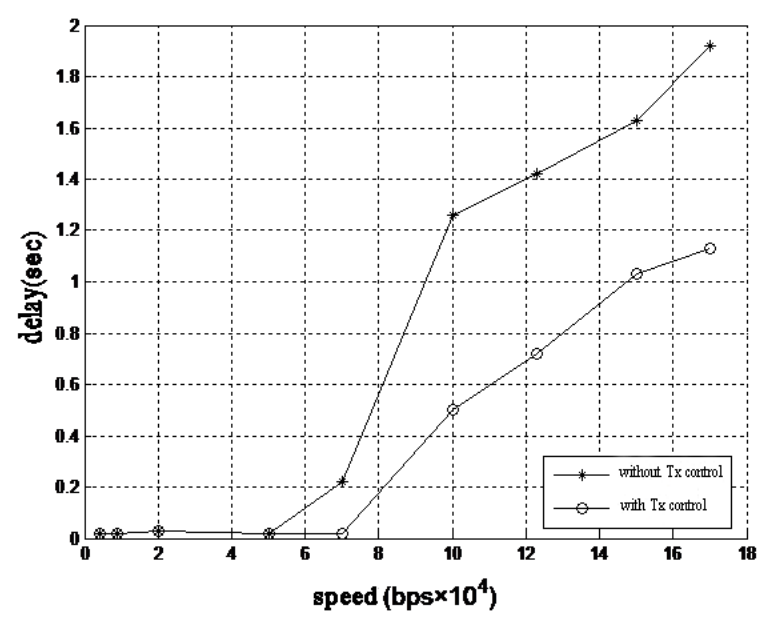

Figure 4. Delays and Speed

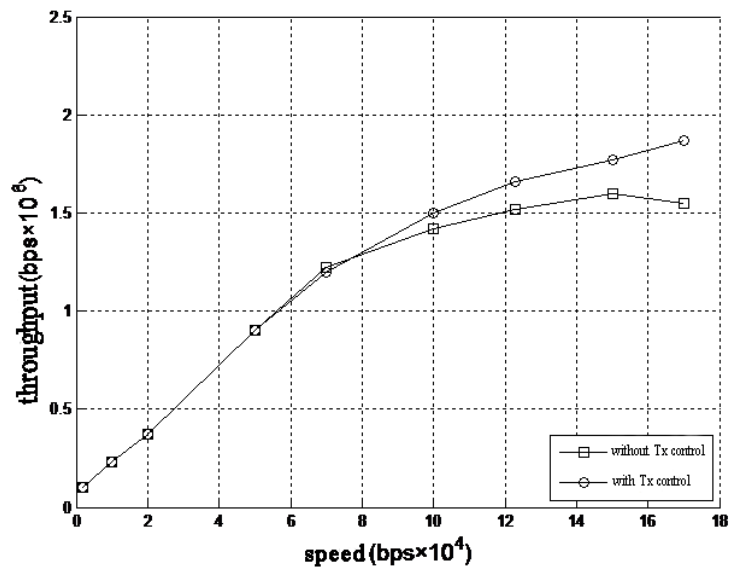

Figure 5. Throughput and Speed

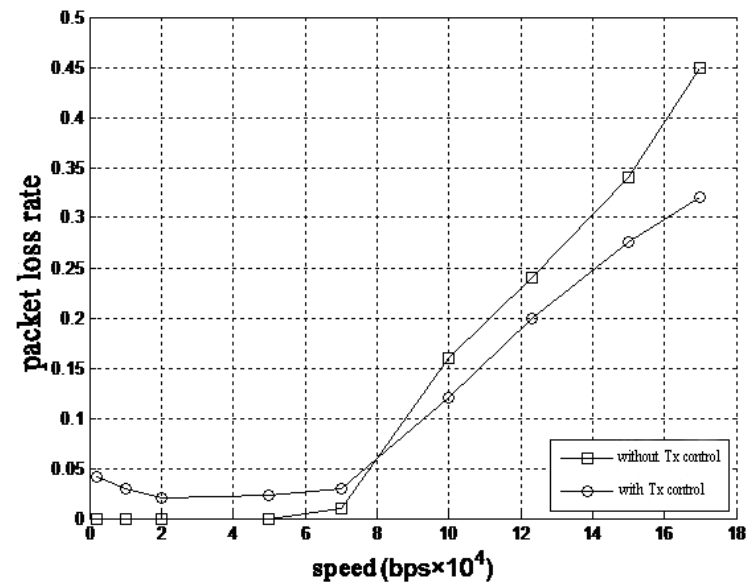

Figure 6. Packet Loss Rate and Speed 


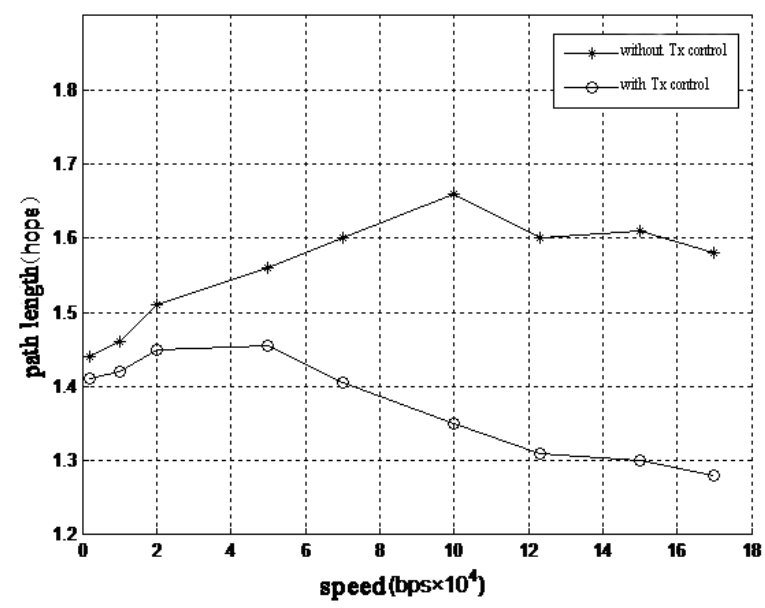

Figure 7. Path Length and Speed

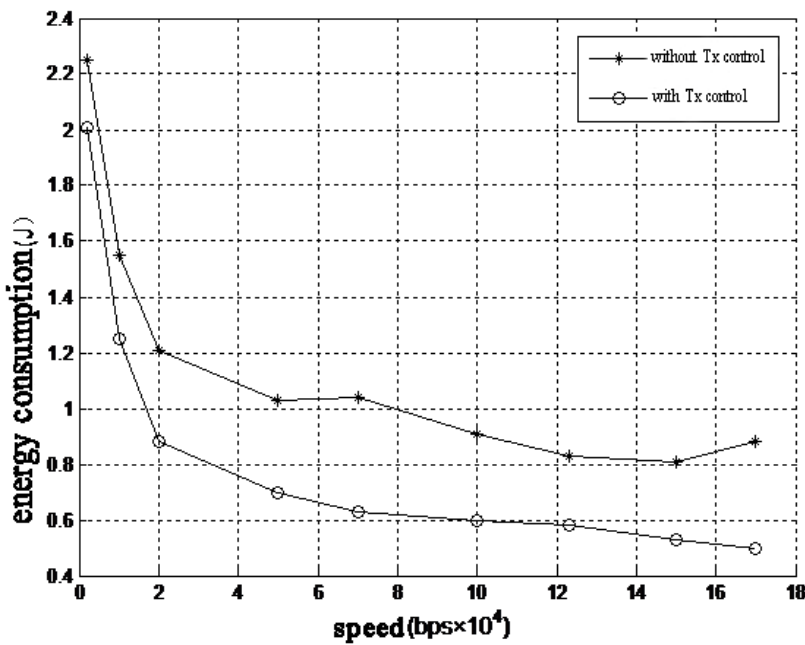

Figure 8. Energy Consumption and Speed

Figure 4 to Figure 8 show the performance improvements of each aspects of the network after the introduction of power control algorithm. From Figure 7, we can see the decrease of path length, which can be explained by "path availability" from [1].

With the application of power control combined with the power-aware AHCPN, the route availability increases, and the speed of nodes' energy consumption slows down; therefore, the usable time of the routes increases.

Simulation shows that when the power assumes a certain value, data flow through the network refers to that which can successfully set up communication links without interfering with the value of neighbor nodes. The data packets will choose node whose remaining battery life is with high probability for data stream transmission.

In this paper, we also show the importance of power control algorithm in CPN protocol: (1) to dynamically discover neighbors and links; (2) to discover and maintain route without much broadcast; (3) to distribute network protocols for the purpose of extend nodes' battery life; (4) to better maintain its performance in comparison with other strategies that are based on broadcast and consumes more energy. 


\section{Conclusions}

In this paper, using a power control algorithm that improves the mobile Ad Hoc network operation based on the previous studies of cognitive packet network (CPN) and AHCPN, the proposed AHCPN protocol is explicated, and its performance is evaluated.

In AHCPN, using full power broadcast SP creates a blooding in part or whole. Though the flooding costs a large amount of energy, SP moves on the network to collect information of neighbor nodes. It is possible that SP can perform unicast transmission based on CPN routing algorithm.

A novel power control strategy that can reduce nodes' MAC competition and energy consumption is proposed in this paper. The simulation of route availability shows the probability of discovering available nodes on the path, which can be improved by lowering nodes', transmit power.

\section{Acknowledgment}

This work was supported by the Scientific Research Project of Colleges and Universities in Gansu Province (No.2013 B-025) and the Youth Science Fund Project of Lanzhou Jiao Tong University (No.2014002).

\section{References}

[1] E. Gelenbe and R. Lent, "Power-Aware Ad hoc Cognitive Packet Networks", IEEE Journal on Selected Areas in Communications, Special Issue on Ad Hoc Networks, vol. 1, (2005).

[2] E. Gelenbe, R. Lent and Z. Xu, "Networks with Cognitive Packets", Opening Key-Note Paper, in: Proceedings of the Eight International Symposium on Modeling, Analysis and Simulation of Computer and Telecommunication Systems (IEEE Computer Society) San Francisco, CA, (2000), pp. 3-12.

[3] E. Gelenbe, R. Lent and Z. Xu, "Toward networks with Cognitive Packets", Opening Invited Paper, International Conference on the Performance of QoS of Next Generation Networking, Nagoya, Japan, in K. Goto, T. Hasegawa, H. Takagi, Y. Takahashi (Eds.), Performance and QoS of Next Generation Networking. Springer, London, (2000).

[4] E. Gelenbe, R. Lent and Z. Xu, "Measurement and Performance of Cognitive Packet Networks", Journal of Computer Networks, vol. 37, (2001), pp. 691-701.

[5] E. Gelenbe, R. Lent and Z. Xu, "Design and Performance of Cognitive Packet Networks", Performance Evaluation, vol. 46, no. 2-3, (2001), pp. 155-176.

[6] E. Royer and C.-K. Toh, "A review of current routing protocols for ad-hoc mobile wireless networks", IEEE Personal Communications.

[7] S. Singh, M. Woo and C. S., "Raghavendra, Power-Aware Routing In Mobile Ad Hoc Networks", Mobile Computing and Networking, Proceedings of The Fourth Annual ACM/IEEE International Conference on Mobile Computing and Networking, (1998), pp. 181-190.

[8] C. K. Toh, "Maximum Battery Life Routing To Support Ubiquitous Mobile Computing In Wireless Ad Hoc Networks", (2001) June.

[9] Q. Li, J. A. Aslam and D. Rus, "Online Power-Aware Routing In Wireless Ad-Hoc Networks", Mobile Computing and Networking, MobiComm 2001, (2001), pp. 97-107.

[10] M. Maleki, K. Dantu and M. Pedram, "Power-Aware Source Routing Protocol For Mobile Ad Hoc Networks", Proceedings of the 2002 International Symposium on Low Power Electronics and Design (2002).

[11] T. Xu, J. Heidemann and D. Estrin, "Adaptive Energy Conserving Routing For Multihop Ad-Hoc Networks”, USC/ISI Research Report 527, (2000) October.

[12] V. Kawadia and P. R. Kumar, "Principles And Protocols For Power Control In Ad Hoc Networks", IEEE Journal on Selected Areas in Communications, Special Issue on Ad Hoc Networks, vol. 1, (2005).

[13] V. Kawadia and P. R. Kumar, "Power Control and Clustering in Ad Hoc Networks", IEEE INFOCOM, (2003).

[14] R. Lent and F. Zonoozi, "Power Control in Ad Hoc Cognitive Packet Networks", Proceedings of the 2005 Texas Wireless Symposium, (2005). 


\section{Author}

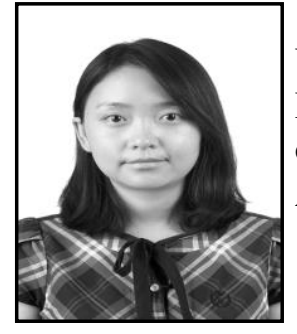

Ting Wang, she received his M.Sc. in Lanzhou Jiao tong University (2008). Now she is full instructor of communication at Internet of Thing Department, University. Since 2013 she is Member of CCF. Her current research interests include different aspects of Artificial Intelligence and Distributed Systems. 
International Journal of Future Generation Communication and Networking Vol. 8, No. 5 (2015) 